\title{
Beta/gamma and alpha backgrounds in CRESST-II Phase 2
}

R. Strauss, ${ }^{a, 1}$ G. Angloher ${ }^{a}$ A. Bento ${ }^{c}$ C. Bucci ${ }^{d}$ L. Canonica ${ }^{d}$ A. Erb $^{b, e}$ F.v. Feilitzsch ${ }^{b}$ N. Ferreiro lachellini ${ }^{a}$ P. Gorla ${ }^{d}$ A. Gütlein ${ }^{f}$

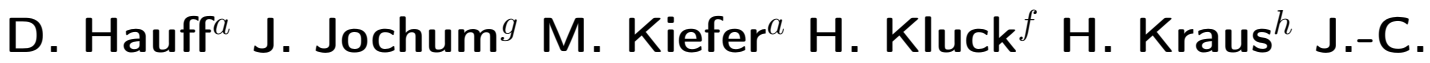
Lanfranchi $^{b}$ J. Loebell ${ }^{g}$ A. Münster ${ }^{b}$ F. Petricca ${ }^{a}$ W. Potzel ${ }^{b}$ F. Pröbst $^{a}$ F. Reindl ${ }^{a}$ S. Roth ${ }^{b}$ K. Rottler ${ }^{g}$ C. Sailer ${ }^{g}$ K. Schäffner ${ }^{d}$ J. Schieck ${ }^{f}$ S. Scholl ${ }^{g}$ S. Schönert ${ }^{b}$ W. Seidel ${ }^{a}$ M.v. Sivers ${ }^{b, 2}$ L. Stodolsky $^{a}$ C. Strandhagen ${ }^{g}$ A. Tanzke ${ }^{a}$ M. Uffinger ${ }^{g}$ A. Ulrich ${ }^{b}$ I. Usherov ${ }^{g}$ S. Wawoczny ${ }^{b}$ M. Willers ${ }^{b}$ M. Wüstrich ${ }^{a}$ A. Zöller ${ }^{b}$

\footnotetext{
${ }^{a}$ Max-Planck-Institut für Physik, D-80805 München, Germany

${ }^{b}$ Physik-Department, Technische Universität München, D-85748 Garching, Germany ${ }^{c}$ CIUC, Departamento de Fisica, Universidade de Coimbra, P3004 516 Coimbra, Portugal ${ }^{d}$ INFN, Laboratori Nazionali del Gran Sasso, I-67010 Assergi, Italy

${ }^{e}$ Walther-Meißner-Institut für Tieftemperaturforschung, D-85748 Garching, Germany ${ }^{f}$ Institut für Hochenergiephysik der Österreichischen Akademie der Wissenschaften, A-1050 Wien, Austria and Atominstitut, Vienna University of Technology, A-1020 Wien, Austria ${ }^{g}$ Physikalisches Institut, Eberhard-Karls-Universität Tübingen, D-72076 Tübingen, Germany ${ }^{h}$ Department of Physics, University of Oxford, Oxford OX1 3RH, United Kingdom

E-mail: strauss@mpp.mpg.de
}

\begin{abstract}
The experiment CRESST-II aims at the detection of dark matter with scintillating $\mathrm{CaWO}_{4}$ crystals operated as cryogenic detectors. Recent results on spin-independent WIMPnucleon scattering from the CRESST-II Phase 2 allowed to probe a new region of parameter space for WIMP masses below $3 \mathrm{GeV} / \mathrm{c}^{2}$. This sensitivity was achieved after background levels were reduced significantly. We present extensive background studies of a $\mathrm{CaWO}_{4}$ crystal, called TUM40, grown at the Technische Universität München. The average beta/gamma rate of $3.51 /[\mathrm{kg} \mathrm{keV}$ day] $(1-40 \mathrm{keV})$ and the total intrinsic alpha activity from natural decay chains of $3.08 \pm 0.04 \mathrm{mBq} / \mathrm{kg}$ are the lowest reported for $\mathrm{CaWO}_{4}$ detectors. Contributions from cosmogenic activation, surface-alpha decays, external radiation and intrinsic alpha/beta emitters are investigated in detail. A Monte-Carlo based background decomposition allows to identify the origin of the majority of beta/gamma events in the energy region relevant for dark matter search.
\end{abstract}

\footnotetext{
${ }^{1}$ Corresponding author.

${ }^{2}$ Present address: Albert Einstein Center for Fundamental Physics, University of Bern, CH-3012 Bern, Switzerland.
} 


\section{Contents}

1 Introduction 1

2 The detector module TUM40 1

3 Experimental results 3

3.1 Beta/gamma background 3

3.2 Surface-alpha background 4

$\begin{array}{lll}3.3 & \text { Alpha background } & 6\end{array}$

4 Monte Carlo based decomposition of the background 8

$\begin{array}{llr}5 & \text { Conclusions and Outlook } & 10\end{array}$

\section{Introduction}

During the last two decades, the sensitivity of experiments aiming at the direct detection of particle dark matter [1] in form of weakly interacting massive particles (WIMPs) [2] has been constantly improved. For the spin-independent WIMP-nucleon cross section impressive sensitivities were reached: currently the liquid-xenon based LUX [3] experiment reports the best upper limit $\left(7.6 \cdot 10^{-10} \mathrm{pb}\right.$ at $\left.33 \mathrm{GeV} / \mathrm{c}^{2}\right)$. A variety of experiments with different techniques [4] have been operated, however, the results are not consistent. A few experiments [5-7], among which is CRESST-II (Cryogenic Rare Event Search with Superconducting Thermometers) [8], reported a signal excess which is not compatible with limits of other dark matter searches [912]. Data from a re-analysis of the commissioning run of CRESST-II [13] showed slight tension with a WIMP interpretation of CRESST-II data and, recently, the first data of CRESST-II Phase $2[8]$ suggest a background origin of the excess.

The improvement in sensitivity of CRESST-II detectors has been achieved by a significant reduction of backgrounds. In this paper, we present a comprehensive study of the different backgrounds observed in CRESST-II Phase 2 (section 3). The knowledge of the background origin is crucial for future dark matter searches based on the CRESST technology. The Monte-Carlo (MC) based decomposition of the spectrum into different background sources which is presented in section 4 gives an important input for future $\mathrm{R} \& \mathrm{D}$ activities.

\section{The detector module TUM40}

CRESST-II detectors are based on a two-channel detector readout which is the key feature to discriminate irreducible radioactive backgrounds. $\mathrm{CaWO}_{4}$ crystals of 200-300 g each, equipped with transition-edge-sensors [14], are operated as cryogenic detectors (called phonon detectors) which allow to measure precisely the total deposited energy $E$ of a particle interaction. An excellent energy threshold of $\mathcal{O}(500 \mathrm{eV})$ and a resolution on a \%olevel (at $2.6 \mathrm{keV}$ ) were achieved $[8,15]$. In addition, the scintillation light output of these crystals is monitored by a cryogenic silicon-on-sapphire detector (called light detector). Since the relative amount of 


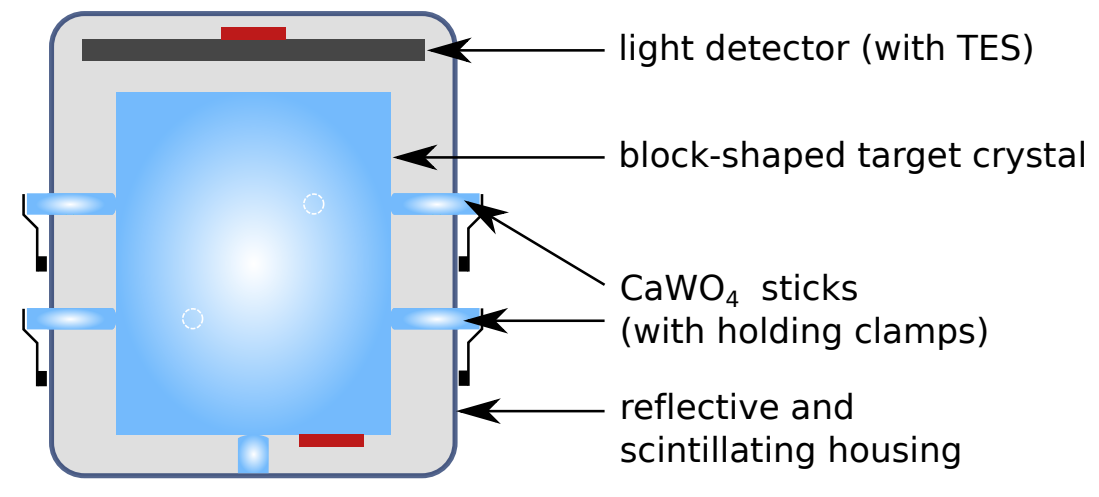

Figure 1. Schematic view of the novel detector module. The block-shaped target crystal TUM40 with a mass of $m=249 \mathrm{~g}$ is held by $\mathrm{CaWO}_{4}$ sticks. Together with the scintillating polymeric foil the sticks establish a fully scintillating inner detector housing which provides an efficient active veto against surface events. A separate silicon-on-sapphire light detector is installed. For details see [15].

scintillation light, called light yield $(L Y)^{1}$, strongly depends on the kind of particle interaction (due to quenching [16]) this channel provides a discrimination of beta/gamma $(L Y \sim 1)$, alpha $(L Y \sim 0.2)$ and nuclear-recoil events $(L Y \lesssim 0.1)$. To a certain extent even $\mathrm{O}$, Ca and $\mathrm{W}$ recoils can be distinguished [17]. CRESST uses a unique multi-element target for WIMP search in a single experiment.

Due to a finite resolution of the light channel, the beta/gamma and nuclear-recoil bands overlap in the region-of-interest (ROI) for dark matter search which is typically defined between energy threshold and $40 \mathrm{keV}$. Several background sources related to surface-alpha decays were identified in the previous run of CRESST-II [18]. In particular recoiling ${ }^{206} \mathrm{~Pb}$ nuclei from ${ }^{210} \mathrm{Po}$ decays were a serious background. These events appear at low light yields similar to $\mathrm{W}$ recoils [15] and might thus be indistinguishable from potential WIMP scatters.

However, the decay of ${ }^{210} \mathrm{Po}$ has a corresponding alpha particle with an energy in the $\mathrm{MeV}$ range which is used to reject this background source. Alphas hitting scintillating materials surrounding the $\mathrm{CaWO}_{4}$ crystals produce sufficient additional light to clearly identify a simultaneously occurring recoil of a heavy nucleus.

The crystal investigated in this paper, a block-shaped $\mathrm{CaWO}_{4}$ crystal of $249 \mathrm{~g}$, is mounted in a novel detector module. Instead of (non-scintillating) metal clamps, $\mathrm{CaWO}_{4}$ sticks are used to support the target crystal. The crystal is surrounded by a reflective and scintillating polymeric foil which together with the $\mathrm{CaWO}_{4}$ sticks realizes a detector housing with fullyscintillating inner surfaces. A schematic view of the novel detector design is shown in Fig. 1, for a detailed description see [15]. As will be presented in section 3.2, this concept provides an efficient rejection of surface-alpha induced Pb-recoils and solves the main background problem of earlier CRESST-II runs [18]. With this detector, a phonon trigger threshold of $\sim 0.60 \mathrm{keV}$ and a resolution of $\sigma=(0.090 \pm 0.010) \mathrm{keV}$ (at $2.60 \mathrm{keV}$ ) were achieved [8, 15].

The detector module presented in this paper uses a $\mathrm{CaWO}_{4}$ crystal, called TUM40, grown at the crystal laboratory of the Technische Universität München (TUM) [19]. At this institute, a Czochralski crystal-production facility was set up, dedicated to the growth of $\mathrm{CaWO}_{4}$. The furnace is exclusively used for the CRESST experiment which is crucial for the requirements in terms of radiopurity. Since radioactive isotopes in $\mathrm{CaWO}_{4}$ crystals can originate from the

\footnotetext{
${ }^{1}$ The light yield is defined as 1 for electron recoils of $122 \mathrm{keV}$, i.e. the light energy of such events is defined as $122 \mathrm{keV}_{\text {ee }}$, the so-called electron-equivalent energy [15].
} 
raw materials themselves [20], a careful selection of these materials is necessary. In particular, in $\mathrm{CaCO}_{3}$ powders which are often extracted from geological settings considerable contaminations were found (e.g. ${ }^{226} \mathrm{Ra}$ with an activity of $\left.\mathcal{O}(10 \mathrm{mBq} / \mathrm{kg})[20]\right)$. Beyond that, impurities can be introduced during processing and handling of raw materials, during crystal growth and detector production. An important source of contamination is Rn-implantation from air. The gaseous isotope ${ }^{222} \mathrm{Rn}$ (half-life $3.6 \mathrm{~d}$ ) which is produced in the ${ }^{238} \mathrm{U}$ chain deposits onto surfaces and the daughter isotopes can be implanted. Therefore, in particular the surfaces of the detectors and of their surroundings get contaminated and the exposure to air containing $\mathrm{Rn}$ has to be minimized. Further, storing CaWO4, which is often delivered as a powder, requires adequate storage to minimize Rn-related backgrounds.

\section{Experimental results}

The first data of TUM40 in CRESST-II Phase 2 with an exposure of $29 \mathrm{~kg}$-days were used for a low-mass WIMP analysis [8] and for the background studies presented in this paper.

\subsection{Beta/gamma background}

The dominant part of events observed with CRESST-II detector modules are beta or gamma induced electron recoils which result in a highly populated beta/gamma band. At lowest energies $(E \lesssim 10 \mathrm{keV})$, due to the finite resolution of the light detector there is a strong overlap with the ROI for dark matter search. Therefore, the beta/gamma background level is crucial for the sensitivity of CRESST-II detectors.

The low-energy spectra $(E \leq 100 \mathrm{keV})$ of commercially available $\mathrm{CaWO}_{4}$ crystals are usually dominated by two intrinsic beta/gamma background components [21]:

- Beta-decays of ${ }^{210} \mathrm{~Pb}$ which is part of the ${ }^{238} \mathrm{U}$ chain. It decays into ${ }^{210} \mathrm{Bi}$ with a half-life of 22.3 years [22]. In $84 \%$ to all cases, an excited energy state of ${ }^{210} \mathrm{Bi}$ with an energy of $46.5 \mathrm{keV}$ is populated. If the contamination is intrinsic to the crystal, both, the energy of the de-excitation gamma and that of the corresponding electron is detected in the crystal. These energy depositions cannot be resolved as separate events with cryogenic detectors and, hence, the two signals add. In many crystals, prominent ${ }^{210} \mathrm{~Pb}$ beta spectra are visible (decreasing in intensity from $46.5 \mathrm{keV}$ towards the Qvalue of $63.5 \mathrm{keV}$ ) which are evidence of strong internal contaminations. If a ${ }^{210} \mathrm{~Pb}$ decay takes place on surfaces of materials outside the detector modules, only the deexcitation gamma is detected which results in a distinctive peak at $46.5 \mathrm{keV}$ (external contamination).

- Beta-decays of ${ }^{227} \mathrm{Ac}$ which is part of the ${ }^{235} \mathrm{U}$ chain. It decays to ${ }^{227} \mathrm{Th}$ with a half-life of 21.8 years [22]. In $35 \%$ (10\%) of the decays, an excited state of the daughter nucleus at the energy level of $24.5 \mathrm{keV}(9.1 \mathrm{keV})$ is populated which relaxes by gamma-emission to the ground state. Therefore, 3 beta-spectra are visible, each of them extending up to the Q-value of $44.8 \mathrm{keV}$ with two characteristic edges at $24.5 \mathrm{keV}$ and $9.1 \mathrm{keV}$ [23].

In Fig. 2, the characteristic features of ${ }^{210} \mathrm{~Pb}$ and ${ }^{227} \mathrm{Ac}$ are clearly visible in the spectrum (black dashed line) of the crystal "VK31" which is operated in CRESST-II Phase 2. The mean background rate in the ROI is about 30 counts/[kg keV day] which is a typical value 
for $\mathrm{CaWO}_{4}$ crystals of this supplier ${ }^{2}$. The best such commercial crystal in terms of intrinsic radiopurity, called "Daisy", has an average rate of $\sim 6$ counts/[kg keV day] (red dashed line in Fig. 2).

For the first time, a detailed beta/gamma-background study of a TUM-grown crystal (TUM40) was performed in this paper. The bulk contamination and the average background rate could significantly be reduced to $3.51 /[\mathrm{kg} \mathrm{keV}$ day $]$ in the ROI which is a reduction by a factor of 2-10 compared to commercial $\mathrm{CaWO}_{4}$ crystals. The histogram in Fig. 2 shows the low-energy spectrum of TUM40 from the first $29 \mathrm{~kg}$-days exposure of CRESST-II Phase 2. Distinct gamma (X-ray) peaks appear above a rather flat background which was not observed in commercial crystals. The dominant ones could be identified to originate from cosmogenic activation of $\mathrm{W}$ isotopes:

- Proton capture on ${ }^{182} \mathrm{~W}$ (and a successive decay) can result in ${ }^{179}$ Ta which decays via EC to ${ }^{179} \mathrm{Hf}$ with a half-life of $665 \mathrm{~d}$. The EC signature in our detectors is exactly the binding energy of the shell electrons of ${ }^{179} \mathrm{Hf}$. In addition to a peak at $65.35 \mathrm{keV}$ (K-shell) which was reported earlier [21] also distinct peaks at $11.27 \mathrm{keV}$ (L1-shell), $10.74 \mathrm{keV}$ (L2-shell) and $2.60 \mathrm{keV}$ (M1-shell) could be identified here. The energies of all identified lines agree within errors with literature values and are listed in Table 1 with their corresponding activities. For the latter, the detection efficiency of TUM40 is considered (see [8]). The K-shell EC line of ${ }^{179} \mathrm{Ta}$ has the highest rate: $A_{K}=(277.2 \pm 15.7) \mu \mathrm{Bq} / \mathrm{kg}$.

- Proton capture on ${ }^{183} \mathrm{~W}$ can result in ${ }^{181} \mathrm{~W}$ which decays via electron capture (EC) to ${ }^{181} \mathrm{Ta}$ with a half-life of $121 \mathrm{~d}$. We confirm the presence of a line at $74.02 \mathrm{keV}$ (K-shell $+6.2 \mathrm{keV}$ gamma) [21] with an activity of $A_{K}=(58.1 \pm 14.5) \mu \mathrm{Bq} / \mathrm{kg}$. To observe higher-order shell EC processes, present statistics is not yet sufficient ${ }^{3}$.

In addition, gamma lines from external radiation can be identified: lines at $46.54 \mathrm{keV}$ from ${ }^{210} \mathrm{~Pb}$ and, at higher energies, from ${ }^{234} \mathrm{Th},{ }^{226} \mathrm{Ra},{ }^{212} \mathrm{~Pb},{ }^{208} \mathrm{Tl},{ }^{214} \mathrm{Bi},{ }^{228} \mathrm{Ac}$ and ${ }^{40} \mathrm{~K}$. A peak from copper X-ray fluorescence at $8.05 \mathrm{keV}\left(\mathrm{K}_{\alpha}\right)$ is observed since most of the material surrounding the detector is copper. The corresponding activities are listed in Table 1 . The contribution of these lines to the background in the ROI is investigated in section 4 .

There are no obvious features of beta spectra visible in the data of TUM40. However, the background contribution of beta/gamma decays from natural decay chains could be derived by a detailed analysis of the alpha spectra (see section 3.3) in combination with a dedicated MC simulation (see section 4).

\subsection{Surface-alpha background}

A more detailed description of the crucial backgrounds related to surface alpha-events is given in [15]. Here, the basic results of that analysis are presented.

The data of CRESST-II detectors is often displayed in the light yield vs. energy plane, as shown in Fig. 3 for TUM40: At light yields of $\sim 1$ the dominant beta/gamma band arises, almost horizontal at energies $\gtrsim 50 \mathrm{keV}$ and slightly decreasing (in light yield) towards smaller energies due to the scintillation properties of $\mathrm{CaWO}_{4}$ [23]. The parametrisation of

\footnotetext{
${ }^{2}$ The crystals were supplied by the General Physics Institute of the Russian Academy of Sciences (Moscow, Russia)

${ }^{3}$ Since CRESST-II Phase 2 is planned to run for $\sim 2$ years a further confirmation of these peaks is in reach by comparing the evolution of the event rate over time with the expected half-lifes.
} 


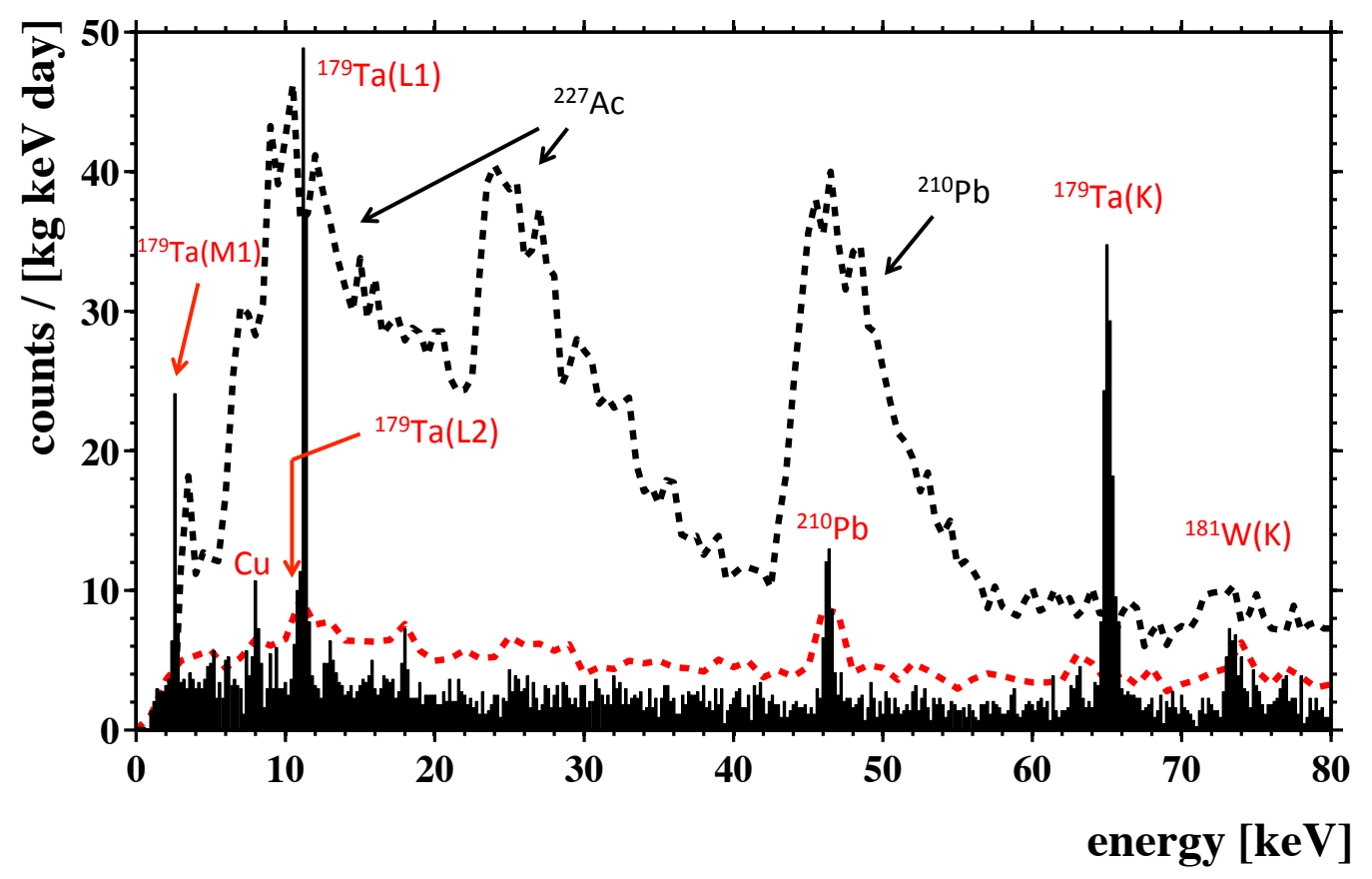

Figure 2. Histogram of the low-energy events (black bars) recorded during CRESST-II Phase 2 with TUM40 which was produced at the Technische Universität München. The most prominent peaks are labelled (in red). In comparison, the background levels of commercial $\mathrm{CaWO}_{4}$ crystals operated as detectors in CRESST II Phase 2 are shown. The dashed red line indicates the rate of the crystal "Daisy" which has the lowest background rate among all commercial crystals $(\sim 6 \mathrm{counts} /[\mathrm{kg} \mathrm{keV}$ day]). The dashed black line shows the background of a typical commercial crystal, called "VK31" $(\sim 30$ counts $/[\mathrm{kg} \mathrm{keV}$ day $])$. In that, the characteristic beta spectra of ${ }^{227} \mathrm{Ac}$ and ${ }^{210} \mathrm{~Pb}$ clearly dominate.

the event bands is given in [15]. The ROI for dark matter search, including all 3 nuclear recoil bands from threshold to $40 \mathrm{keV}^{4}$, is shown as a grey area in Fig. 3. The reference region for ${ }^{206} \mathrm{~Pb}$ recoils induced by ${ }^{210} \mathrm{Po}$ alpha decays is also indicated in the plot (area enclosed by the green line) at light yields of $\sim 0.01$ between $40 \mathrm{keV}$ and $107 \mathrm{keV}$. In earlier runs of CRESST-II this region was populated: At the full kinetic energy of the $\mathrm{Pb}$ nucleus (decay on surfaces of surrounding material and of the crystal) a peak arose at $\sim 103 \mathrm{keV}$ with a exponential tail towards lower energies. The latter comes from ${ }^{210} \mathrm{Po}$ decays which are implanted in surrounding material. It was identified to originate from a contamination in the metal clamps holding the crystals (e.g. by Rn-implantation from air). The resulting spectrum of Pb-recoils was reproduced by a SRIM simulation [18].

In the first data of TUM40 acquired in CRESST-II Phase 2 no events are observed in the reference region for ${ }^{206} \mathrm{~Pb}$ recoils, while with the background level of Phase 1 [18] $6.9 \pm 2.6$ events would be expected. Since in the novel detector module (see section 2) the crystal is completely surrounded by scintillating materials, the corresponding alpha with an energy of $\sim 5.3 \mathrm{MeV}$ produces sufficient additional light to shift ${ }^{206} \mathrm{~Pb}$ recoils out of the nuclear recoil region. A population of such vetoed events is visible in Fig. 3 at light yields of 0.2-0.5 (red dots). This first data show the high efficiency of the surface-alpha event rejection realized

\footnotetext{
${ }^{4}$ In [8], the $50 \%$ acceptance bound for O recoils is defined as the upper light yield bound for the ROI. This convention is also used in this paper.
} 
Table 1. Activities $A$ of the identified gamma-lines measured with the crystal TUM40 in $29 \mathrm{~kg}$ days of exposure during CRESST-II Phase 2. External gamma lines (ext.) as well as peaks from cosmogenically-induced EC decays are listed (the shell of the captured electron is shown in brackets).

\begin{tabular}{lcc}
\hline source & $E_{\text {lit }}[\mathrm{keV}][22]$ & $A[\mu \mathrm{Bq} / \mathrm{kg}]$ \\
\hline${ }^{179} \mathrm{Ta}$ (M1) & 2.6009 & $70.3 \pm 15.8$ \\
$\mathrm{Cu}$ X-ray (ext.) & 8.048 & $27.1 \pm 14.3$ \\
${ }^{179} \mathrm{Ta}$ (L2) & 10.74 & $24.8 \pm 14.5$ \\
${ }^{179} \mathrm{Ta}$ (L1) & 11.271 & $202.2 \pm 16.0$ \\
${ }^{210} \mathrm{~Pb}$ (ext.) & 46.54 & $78.6 \pm 14.8$ \\
${ }^{179} \mathrm{Ta}$ (K) & 65.35 & $277.2 \pm 15.7$ \\
${ }^{181} \mathrm{~W}$ (K) & 74.02 & $58.1 \pm 14.5$ \\
${ }^{234} \mathrm{Th}$ (ext.) & 92.4 & $123.5 \pm 11.7$ \\
${ }^{226} \mathrm{Ra}$ (ext.) & 186.2 & $109.0 \pm 11.2$ \\
${ }^{212} \mathrm{~Pb}$ (ext.) & 238.6 & $185.6 \pm 12.9$ \\
${ }^{208} \mathrm{Tl}$ (ext.) & 583.2 & $93.4 \pm 8.4$ \\
${ }^{214} \mathrm{Bi}$ (ext.) & 609.2 & $69.3 \pm 24.6$ \\
${ }^{228} \mathrm{Ac}$ (ext.) & 911.2 & $54.8 \pm 6.8$ \\
${ }^{228} \mathrm{Ac}$ (ext.) & 969.0 & $36.3 \pm 6.1$ \\
${ }^{40} \mathrm{~K}$ (ext.) & 1432.7 & $41.4 \pm 6.2$ \\
${ }^{208} \mathrm{Tl}$ (ext.) & 2614.0 & $28.5 \pm 3.9$ \\
\hline
\end{tabular}

with the new detector concept. No such backgrounds are expected within the sensitivity goals of CRESST-II Phase 2 with the final exposure [15].

Furthermore, an additional background source most probably originating from contaminations on surfaces of materials outside the detector housing was identified in this paper. Electrons (e.g. from beta-emitters from natural decay chains) pass through the polymeric foil (thickness $50 \mu \mathrm{m}$ ), lose some of their energy therein and produce additional light before getting absorbed by the $\mathrm{CaWO}_{4}$ crystal. Dedicated measurements show that electrons, independent of their energy, produce a roughly fixed amount of scintillation light in the foil with an energy of $\mathcal{O}(1 \mathrm{keV})$. This explains the additional population of events above the beta/gamma band (labelled by "external betas" in Fig. 3). Since an approximately fixed amount of addition scintillation light adds to the light produced by the beta in the crystal, the excess in light yield gets bigger towards lower energies. The analysis performed in section 4 finds a significant contribution of this event population to the overall background in the ROI.

\subsection{Alpha background}

Due to the phonon-light technique of CRESST-II detectors, alpha events can be perfectly discriminated from other backgrounds, particularly, from the dominant beta/gamma populations. In case of an internal contamination, the full energy (Q-value) of a decay is measured. Therefore, the most obvious approach to investigate intrinsic radioactivity of $\mathrm{CaWO}_{4}$ crystals is the analysis of discrete alpha lines which arise at energies $\gtrsim 2 \mathrm{MeV}$ far off the ROI. Distinct alpha peaks from intrinsic contamination of the natural decay chains as well as of radioactive rare-earth metals (e.g. ${ }^{147} \mathrm{Sm}$ ) and the isotope ${ }^{180} \mathrm{~W}$ are observed.

In TUM40 decays from the three natural decay chains ${ }^{238} \mathrm{U},{ }^{235} \mathrm{U}$ and ${ }^{232} \mathrm{Th}$ are observed. All individual decays are listed with their respective half-life and Q-values $\left(E_{l i t}\right)$ in Table 2. The 


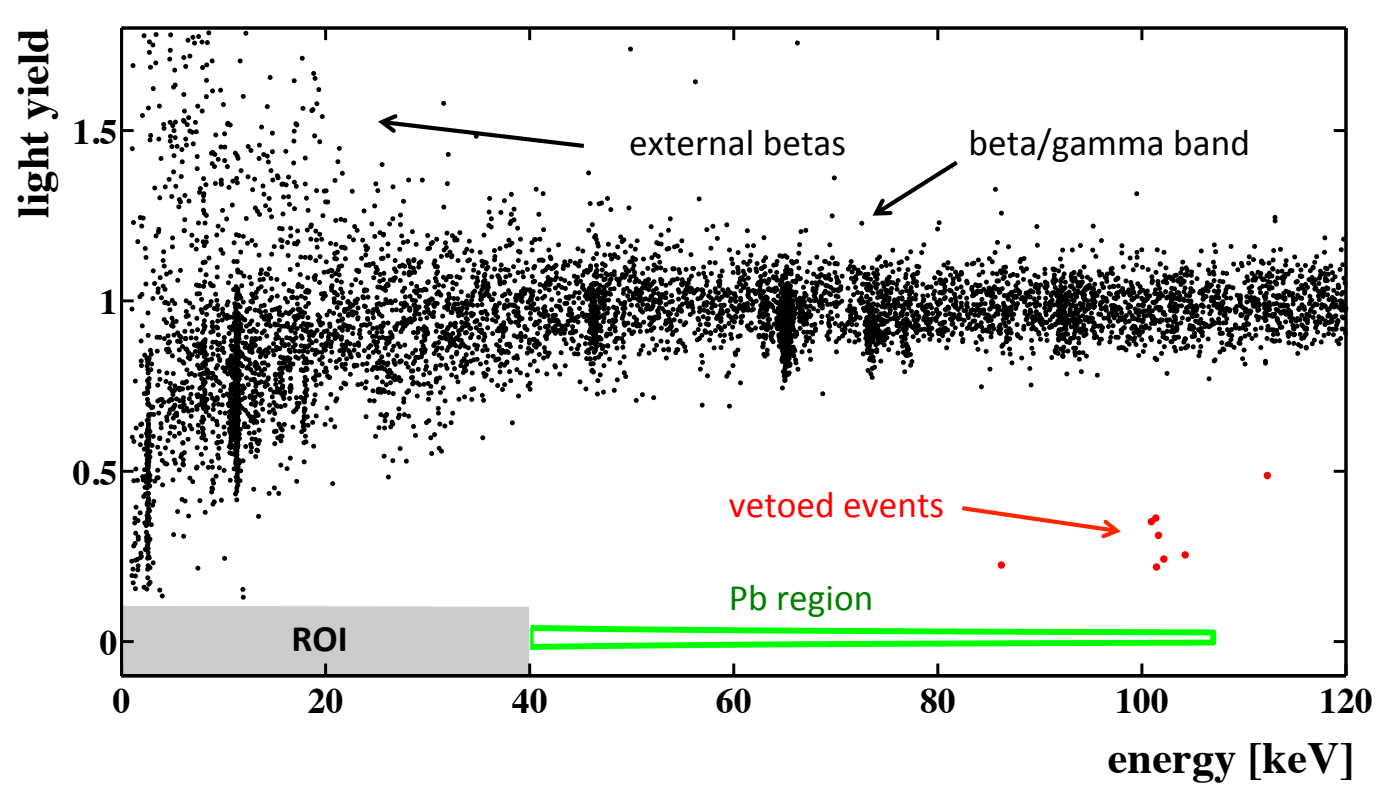

Figure 3. Light yield vs. energy plot of the events (dots) recorded with TUM40 in CRESST-II Phase 2. At light yields of $\sim 1$ the dominant band of beta/gamma events shows up. Due to light quenching [16] nuclear recoils have a reduced light yield. The ROI for dark matter search is shown in grey. Surface-alpha related ${ }^{206} \mathrm{~Pb}$ recoil background would be expected in the region enclosed by the green line. Due to the scintillation veto of the novel detector module, this area is free of events. All ${ }^{206} \mathrm{~Pb}$ recoils observed are shifted to higher light yields (red dots). External betas passing through the scintillating foil show up at higher light yields compared to events in the beta/gamma band.

detailed study of the intrinsic alpha contaminations performed in this work enables to derive all relevant beta-decay rates. This provides a quantification of the intrinsic contamination at low-energies for the first time.

The large dynamic range and the discrimination capability of CRESST-II detectors for alpha events is illustrated in Fig. 4. The energy measured in the phonon detector is plotted versus the (electron-equivalent) energy measured in the light detector with the module TUM40 in $29 \mathrm{~kg}$-days of exposure. Due to light quenching the alpha band is well-separated from the beta/gamma band. Besides the lines of ${ }^{147} \mathrm{Sm}$ and ${ }^{180} \mathrm{~W}$, all peaks can be attributed to alpha decays from natural decay chains clustering at energies between 4 and $7 \mathrm{MeV}$. The total alpha activity of intrinsic contamination is $A_{t o t}=(3.08 \pm 0.04) \mathrm{mBq} / \mathrm{kg}$ to which ${ }^{238} \mathrm{U}$ $((1.01 \pm 0.02) \mathrm{mBq} / \mathrm{kg})$ and ${ }^{234} \mathrm{U}((1.08 \pm 0.03) \mathrm{mBq} / \mathrm{kg})$ from the ${ }^{238} \mathrm{U}$ chain are the dominant contribution. The histogram in Fig. 5 shows the identified alpha peaks of lower intensities $((\sim 10-100) \mu \mathrm{Bq} / \mathrm{kg})$ between 4 and $7 \mathrm{MeV}$. At even higher energies beta-alpha coincident events are visible in Fig. $4\left({ }^{214} \mathrm{Bi} /{ }^{214} \mathrm{Po}\right.$ and ${ }^{212} \mathrm{Bi} /{ }^{212} \mathrm{Po}$ at $\left.\sim 7.8-12 \mathrm{MeV}\right)$. These subsequent decays are too fast to be disentangled as separate events, hence both signals sum up and characteristic continuous bands arise (see [20] for details). Similarly, the alpha-alpha coincidence ${ }^{219} \mathrm{Rn} /{ }^{215} \mathrm{Po}$ shows up at the combined energy of $\sim 14.9 \mathrm{MeV}$. The measured ${ }^{180} \mathrm{~W}$ activity of $(36 \pm 9) \mu \mathrm{Bq} / \mathrm{kg}$ agrees well with the rate of $(31 \pm 8) \mu \mathrm{Bq} / \mathrm{kg}$ obtained by a previous measurement [24] which cross-checks the analysis performed.

All alpha particles originating from natural decay chains are observed in this data and are listed in Table 2 with their individual activities. All alpha decays, short-lived with respect 


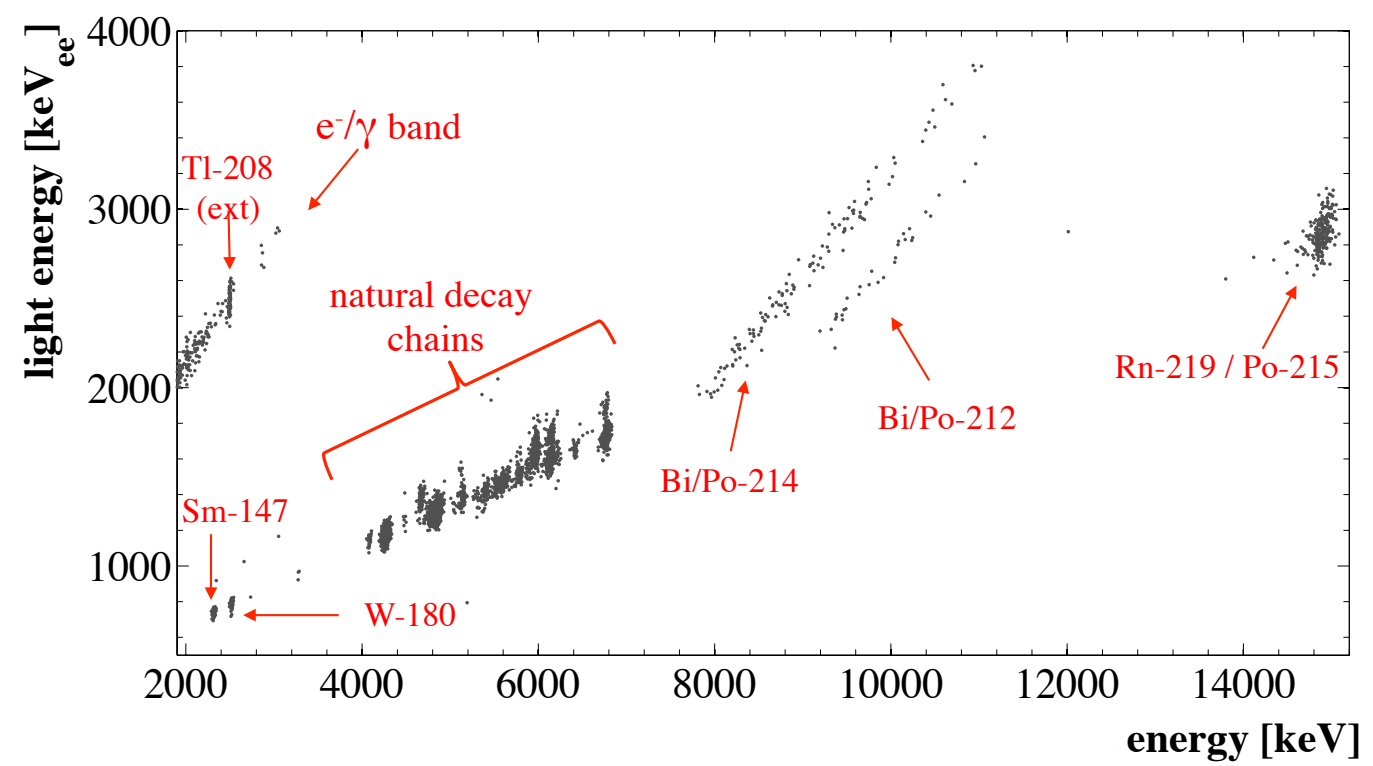

Figure 4. Energy measured in the phonon detector plotted against the energy detected in the light detector in units of electron-equivalent energy $\left(\mathrm{keV}_{e e}\right)$. The alpha lines are well separated from the beta/gamma band due to light quenching $\left(Q F_{\alpha} \sim 0.22\right)$. Most of the discrete alpha lines of the natural decay chains have energies between 4 and $7 \mathrm{MeV}$ (see Fig. 5) except the $\alpha-\alpha\left({ }^{219} \mathrm{Rn} /{ }^{215} \mathrm{Po}\right)$ and $\alpha-\beta\left({ }^{214} \mathrm{Bi} /{ }^{214} \mathrm{Po},{ }^{212} \mathrm{Bi} /{ }^{212} \mathrm{Po}\right)$ coincidences at higher energies. Lines from intrinsic ${ }^{180} \mathrm{~W}$ and a contamination of ${ }^{147} \mathrm{Sm}$ are also visible.

to the measuring time $(\sim 0.5 \mathrm{y})$ agree in rate as expected. The equilibrium of the ${ }^{238} \mathrm{U}$ chain is broken at the long-lived isotopes ${ }^{234} \mathrm{U}^{5}$ and that of the ${ }^{235} \mathrm{U}$ chain at ${ }^{231} \mathrm{~Pa}$. This can originate, e.g., from chemical separation processes during the production steps. All measured intensities are consistent with the individual decay chains (a reduced detection efficiency for the decays ${ }^{220} \mathrm{Rn}$ and ${ }^{216} \mathrm{Po}$ due to pile-up effects has to be considered, for details see [20]).

\section{Monte Carlo based decomposition of the background}

By the detailed alpha analysis presented in section 3.3 the intensities of all beta decays from the natural decay chains ${ }^{238} \mathrm{U},{ }^{235} \mathrm{U}$ and ${ }^{232} \mathrm{Th}$ can be derived since they are in equilibrium with at least one alpha decay (see Table 2). These measured activities were used as input for a dedicated GEANT4 MC simulation (version 10.1) $[25,26]$ which allows to derive the beta/gamma spectra in the ROI for dark matter search. To model the production, propagation, and absorption of beta/gamma particles in the detector down to $250 \mathrm{eV}$, the Livermore low-energy electromagnetic models were used. The electromagnetic model implements photoelectric effect, Compton scattering, Rayleigh scattering, conversion in $\mathrm{e}^{+} \mathrm{e}^{-}$pairs, ionisation, and bremsstrahlung production.

The results of this data-based simulation is shown in Fig. 6 (inset): The blue curve shows the sum of all beta/gamma events from natural decay chains. The 1- $\sigma$ error band (light blue) is a combination of the statistical error of the simulation and the uncertainty of the experimentally determined activities of the beta emitters. This contribution has an activity

\footnotetext{
${ }^{5} \mathrm{~A}$ broken equilibrium at ${ }^{210} \mathrm{~Pb}$ and ${ }^{210} \mathrm{Po}\left({ }^{238} \mathrm{U}\right.$ chain $)$ is possible, however, can not be proven here due to limited statistics.
} 


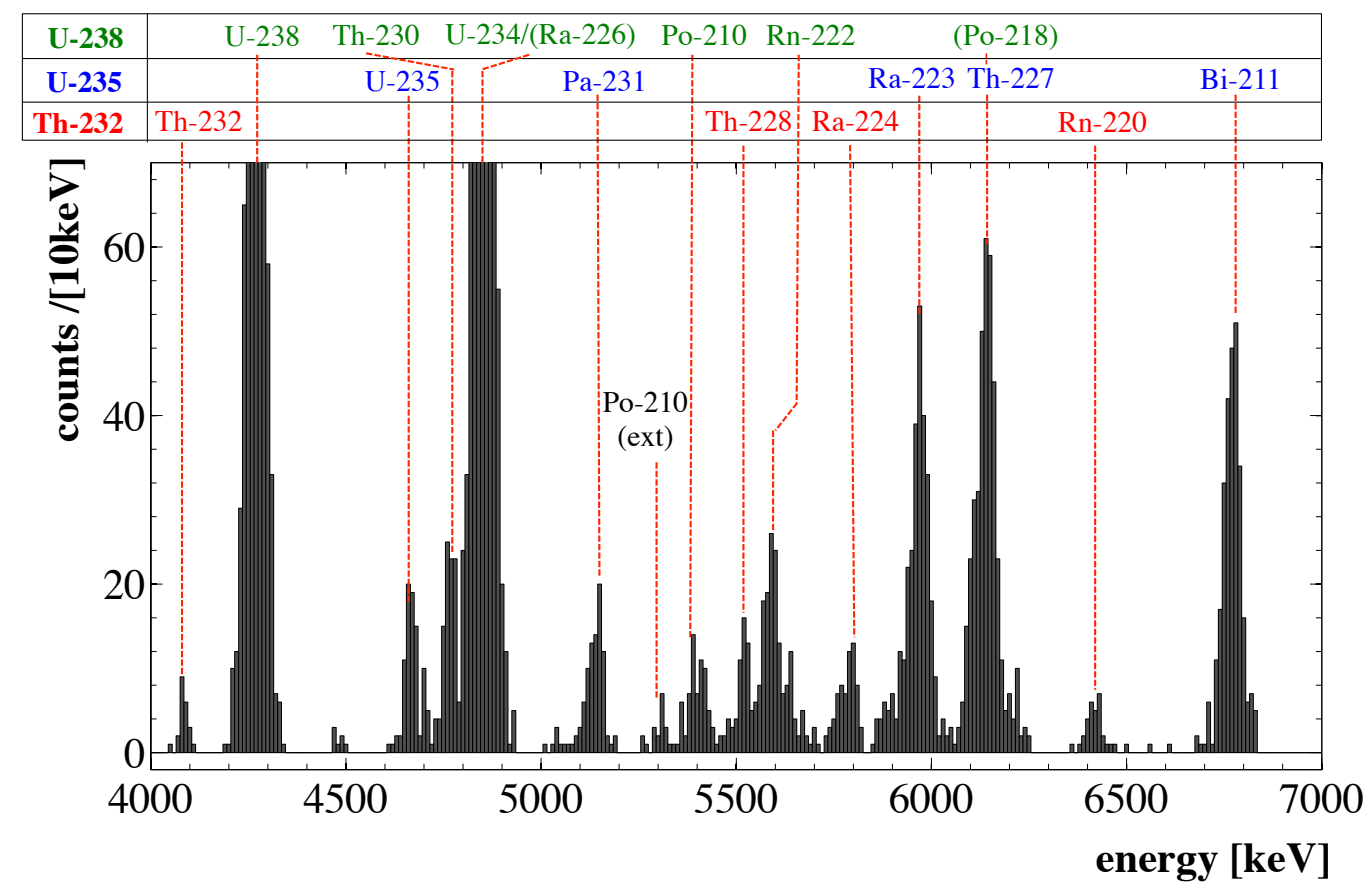

Figure 5. Discrete alpha lines from the natural decay chains observed with the crystal TUM40 in an exposure of $29 \mathrm{~kg}$-days between 4 and $7 \mathrm{MeV}$. The lines identified in the ${ }^{238} \mathrm{U},{ }^{235} \mathrm{U}$ and ${ }^{232} \mathrm{Th}$ chains are listed. In addition, an external ${ }^{210} \mathrm{Po}$ line is visible where only the alpha energy $(5.30 \mathrm{MeV})$ and not the recoil of ${ }^{206} \mathrm{~Pb}(103 \mathrm{keV})$ is detected. The individual activities are listed in Table 2. The peaks of ${ }^{238} \mathrm{U}$ and ${ }^{234} \mathrm{U}$ completely dominate the spectrum (upper cut in histogram at 70 counts/[10 keV]).

of $A_{1-40}=494.2 \pm 48.4 \mu \mathrm{Bq} / \mathrm{kg}$ in the ROI which corresponds to a mean rate of $3.51 \pm 0.09$ counts/[kg keV day]. For the first time, the contribution of the intrinsic beta/gamma emitter could be disentangled. It corresponds to $(30.4 \pm 2.9) \%$ of the total events observed. The main contributions originate from ${ }^{234} \mathrm{Th}(346 \mu \mathrm{Bq} / \mathrm{kg}),{ }^{227} \mathrm{Ac}(93 \mu \mathrm{Bq} / \mathrm{kg}),{ }^{234} \mathrm{~Pa}(35 \mu \mathrm{Bq} / \mathrm{kg})$ and ${ }^{228} \mathrm{Ra}$ decays $(9 \mu \mathrm{Bq} / \mathrm{kg})$. The characteristic edges at $\sim 9 \mathrm{keV}$ and $\sim 24 \mathrm{keV}$ originate from the contribution of the ${ }^{227}$ Ac spectrum (see section 3.1). The values of all relevant beta emitters are listed in Table 3.

Furthermore, the response of the detector to external gamma radiation is studied with a dedicated MC simulation. The intensity of the individual components is scaled such to match the observed external gamma peaks (see section 3.1). All identified external gamma lines which are listed in Table 1 are included in the study. The result is shown as in Fig. 6 (inset) as a green line with the corresponding 1- $\sigma$ error band (light green). The only peak in the ROI identified as to originate from external radiation is the $\mathrm{Cu}$ X-ray peak at $8.0 \mathrm{keV}$. The continuous Compton background from external sources (peaks at higher energies) is negligible in the ROI ( $\lesssim 0.03$ events $/\left[\mathrm{kg} \mathrm{keV}\right.$ day]), however, W-escape peaks originating from the ${ }^{234} \mathrm{Th}$ line at around $92.4 \mathrm{keV}$ contribute significantly $((62.2 \pm 7.8) \mu \mathrm{Bq} / \mathrm{kg})$. X-rays from $\mathrm{W}\left(\mathrm{K}_{\alpha 1}\right.$ : $59.3 \mathrm{keV}, \mathrm{K}_{\alpha 2}: 58.0 \mathrm{keV}, \mathrm{K}_{\beta 1}: 67.2 \mathrm{keV}$ and $\mathrm{K}_{\beta 2}: 69.1 \mathrm{keV}$ [22]) can escape the $\mathrm{CaWO}_{4}$ crystal giving rise to 4 distinct escape peaks between 23 and $35 \mathrm{keV}$. The statistics of the recorded data is not yet sufficient to resolve these peaks, but as shown below, the total observed spectrum is compatible with this prediction.

In addition to that, a background component from external betas has been identified within 
this work. As explained in section 3.2, electrons passing through the polymeric foil produce additional scintillation light before hitting the $\mathrm{CaWO}_{4}$ crystal which shifts these events to higher light yields (see Fig. 3). A phenomenological model to account for this event population was developed in [27]. Measurements show that these events can be nicely described by an exponential distribution decreasing towards higher energies and higher light yields. To obtain the observed event distribution, the spectrum has to be convolved with the resolution of the light measurement. A detailed description of the likelihood fit can be found in the literature $[15,27]$. The result of the likelihood fit to the data of TUM40 is shown in Fig. 6 (inset): The grey curve accounts for the rate of external betas most probably originating from beta emitters on surfaces surrounding the detectors. The corresponding 1- $\sigma$ error bounds are depicted in light grey. An activity of $(274 \pm 137.6) \mu \mathrm{Bq} / \mathrm{kg}$ which corresponds to $(16.9 \pm 8.4) \%$ of the total rate is attributed to external betas.

The EC-peaks (at $2.60 \mathrm{keV}, 10.74 \mathrm{keV}$ and $11.27 \mathrm{keV}$ ) originating from cosmogenic activation of $\mathrm{W}$ isotopes (see section 3.1) account for $(290.7 \pm 17.0) \mu \mathrm{Bq} / \mathrm{kg}((17.9 \pm 1.0) \%$ of the total rate).

The red curve in Fig. 6 (main frame and inset) shows the combined spectrum of all identified backgrounds. The inset shows the corresponding 1- $\sigma$ error bounds (light red). A total activity of $(1121.8 \pm 146.6) \mu \mathrm{Bq} / \mathrm{kg}$ is found which explains $(69.0 \pm 9.0) \%$ of the data. While at energies above $\sim 20 \mathrm{keV}$ the sum of identified sources almost completely reproduces the data, at lower energies a significant part of the spectrum remains unexplained. There are slight hints for additional gamma peaks (e.g at $\sim 18 \mathrm{keV}$ ), for the identification of which, however, more statistics is necessary.

To investigate the relevance of the individual background components for dark matter search, their leakage into the nuclear-recoil bands is calculated based on the background studies developed in this work. The number of events leaking into the ROI (to below the $50 \%$ acceptance bound for O-recoils [8]) is calculated for the beta/gamma spectrum from natural decay chains, external gammas, the cosmogenic activation lines and the contribution of external betas. In Table 3 the cumulative leakage of the individual components into the ROI is listed for an analysis threshold of $1 \mathrm{keV}\left(L_{1}\right)$ and $12 \mathrm{keV}\left(L_{12}\right)$. The cumulative leakage is graphically illustrated in Fig. 7 as a function of the analysis threshold. For an analysis threshold of $1 \mathrm{keV}$ a leakage of $\mathcal{O}\left(10^{2}\right)$ events per detector and year is expected. Here the contaminations due to cosmogenic activation give the highest contribution. Above $\sim 12 \mathrm{keV}$, the leakage drops by $\sim 2$ orders of magnitude and is then dominated by intrinsic beta/gamma events. The calculation shows that an experiment with a threshold of $12 \mathrm{keV}$ would be background-free up to an exposure of $\sim 3$ ton-years.

\section{Conclusions and Outlook}

TUM40 operated in the new detector housing has reached unprecedented background levels. Using $\mathrm{CaWO}_{4}$ sticks to hold the target crystal, a fully-scintillating inner detector housing is realized and backgrounds from surface-alpha decays are rejected with high efficiency. A phonon trigger threshold of $\sim 0.60 \mathrm{keV}$ and a resolution of $\sigma=(0.090 \pm 0.010) \mathrm{keV}$ (at $2.60 \mathrm{keV}$ ) are reached with TUM40. By using a $\mathrm{CaWO}_{4}$ crystal produced at the TUM, the intrinsic background rate was reduced to the lowest level reported for CRESST $\mathrm{CaWO}_{4}$ detectors: on average $3.51 \pm 0.09$ beta/gamma events per $\mathrm{kg} \mathrm{keV}$ day in the ROI (1-40 keV) and a total alpha activity from natural decay chains of $A_{t o t, \alpha}=3.08 \pm 0.04 \mathrm{mBq} / \mathrm{kg}$. In this paper, a detailed alpha analysis was performed which allowed to derive the activities of all decaying 


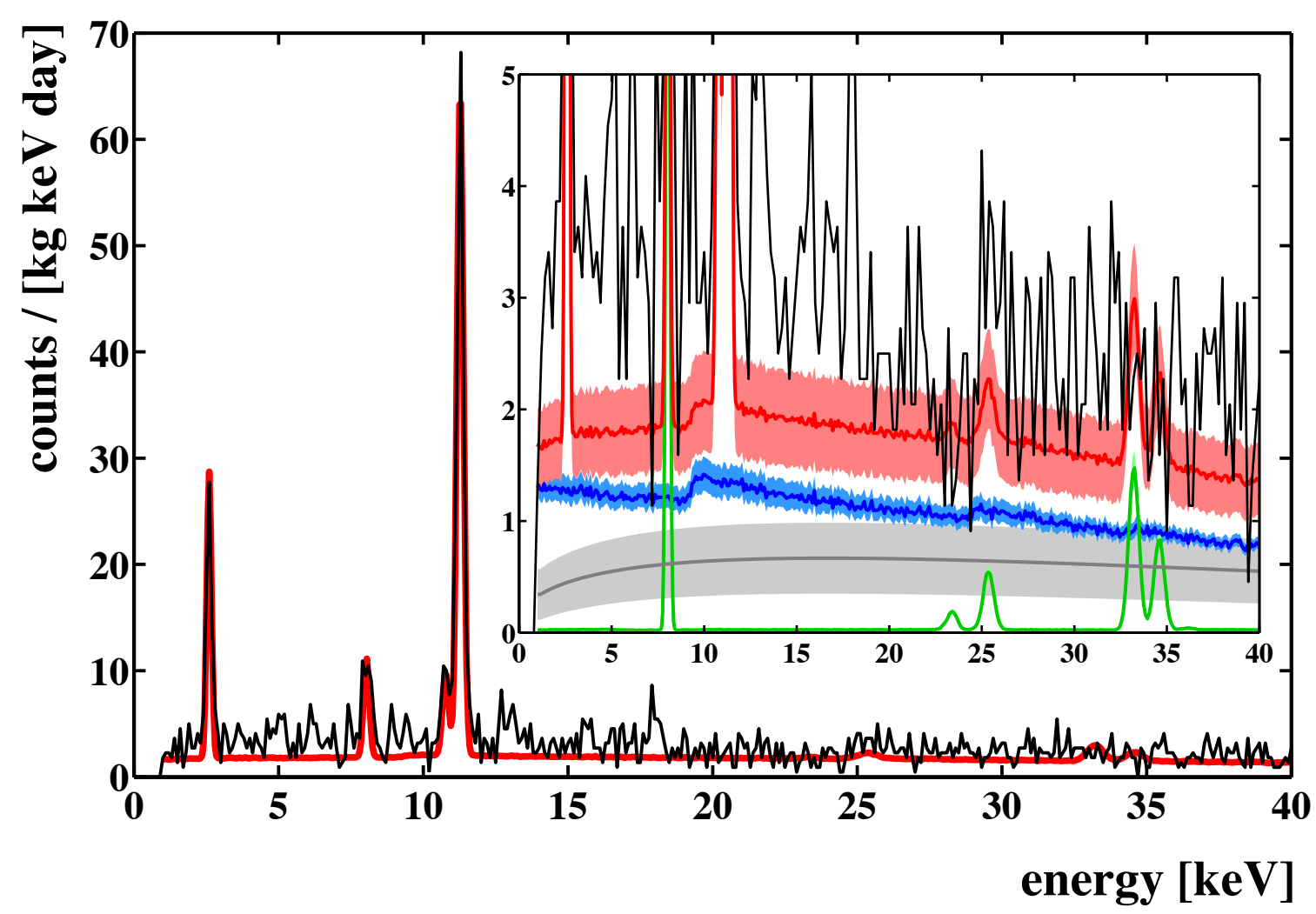

Figure 6. Histogram of the events in the ROI (black line) recorded with TUM40 in CRESST-II Phase 2. The red line indicates the sum of all identified background sources with the dominant peaks from cosmogenic activation $(2.6 \mathrm{keV}, 10.7 \mathrm{keV}, 11.3 \mathrm{keV})$ and the $\mathrm{Cu}$ X-ray line $(8.0 \mathrm{keV})$. Inset: Decomposition of the background based on MC simulation (see text). The contributions of external gamma radiation (green), external betas (grey) and intrinsic beta/gamma radiation from natural decay chains (blue) are shown. The sum of these components (plus gamma peaks) are shown in red. The individual $1-\sigma$ error bands are depicted in the corresponding colour. The identified backgrounds explain $\sim 70 \%$ of the observed events.

isotopes of the natural decay chains. Based on these results, a GEANT4 MC simulation was set up to investigate the contribution of intrinsic beta/gamma backgrounds in the ROI for dark matter search $(1-40 \mathrm{keV})$. An activity of $\sim 494 \mu \mathrm{Bq} / \mathrm{kg}$ was found which corresponds to $\sim 30 \%$ of the total event rate. The MC simulation also shows the contribution of events originating from external gamma radiation. An activity of $62.2 \mu \mathrm{Bq} / \mathrm{kg}(\sim 4 \%$ of total $)$ is found in the ROI. Furthermore, the study shows that $17 \%$ of the background most probably is due to electrons originating from surfaces surrounding the detector and passing through the scintillating housing. Distinct gamma lines are observed in the low-energy spectrum at energies $<80 \mathrm{keV}$, originating from cosmogenic activation of $\mathrm{W}$ isotopes. The isotopes ${ }^{182} \mathrm{~W}$ and ${ }^{183} \mathrm{~W}$, and subsequent EC-reactions of ${ }^{179} \mathrm{Ta}$ and ${ }^{181} \mathrm{~W}$ are identified as sources. The resulting activity in the $\mathrm{ROI}$ is $290.7 \mu \mathrm{Bq} / \mathrm{kg}$ ( $\sim 18 \%$ of the total event rate).

For the first time, the background in the ROI of a CRESST-II detector module could be decomposed in a comprehensive way. The analysis performed suggests that the background of TUM40 is - unlike previously assumed - not exclusively limited by intrinsic contaminations from natural decay chains, but cosmogenic activation and external sources play an important role. The knowledge of the background origin is crucial for the future R\&D activities of 


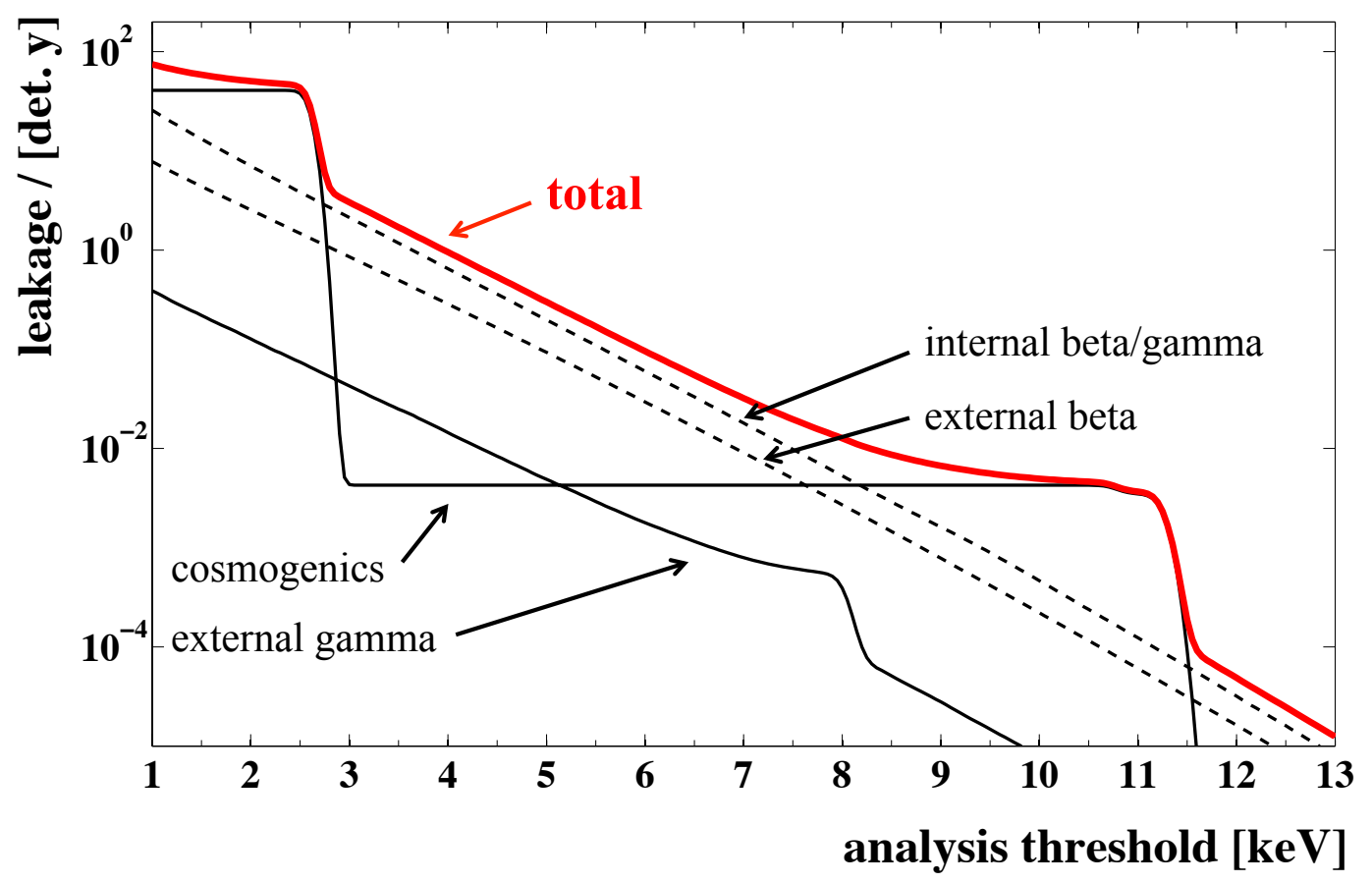

Figure 7. Expected cumulative leakage from the beta/gamma band into the ROI (1 to 40keV) plotted against the analysis threshold. The calculation is based on the background level of the detector TUM40 in CRESST-II Phase 2. The decomposition of the background into individual components is explained in detail in section 4.

CRESST concerning background reduction.

The sensitivity of CRESST-II detectors for dark matter search was investigated by calculating the leakage of beta/gamma events into the ROI. For analysis thresholds $>12 \mathrm{keV}$ the present background level results in a total expected leakage of $<10^{-4}$ events per detector and year. This suggests that even for a ton-scale experiment and typical measuring times of $\mathcal{O}(1$ year $)$ such detectors would not be limited by beta/gamma leakage and, if no additional background sources contribute, would be background-free for high WIMP-mass searches $\left(\gtrsim 5 \mathrm{GeV} / \mathrm{c}^{2}\right)$. The goals of the future EURECA experiment [28], namely to reach the level of $10^{-10} \mathrm{pb}$ for the spin-independent WIMP-nucleon scattering cross-section (for WIMP masses of $\sim 50 \mathrm{GeV} / c^{2}$ ), can be achieved by TUM40-type detectors using state-of-the-art CRESST technology.

Due to the low thresholds achieved, CRESST detector are most suited for the detection of low-mass WIMPs $\left(\lesssim 5 \mathrm{GeV} / \mathrm{c}^{2}\right)$. The potential has been recently demonstrated by the first data of TUM40 in CRESST-II Phase 2 which sets stringent limits on the WIMP-nucleon scattering cross-section and explores new parameter space below WIMP masses of $3 \mathrm{GeV} / \mathrm{c}^{2}$ [8]. The study performed in this paper shows that for an analysis threshold of $1 \mathrm{keV}$ a total leakage of $\sim 10^{2}$ events is expected per detector and year. This agrees nicely with the number of events observed in the ROI during the first $29 \mathrm{~kg}$-days of data acquired with TUM40 and suggests that no other background sources contribute (as e.g. neutrons). To achieve the future sensitivity goals for low-mass WIMP search [29], background levels must be further reduced. External radiation can be diminished by cleaning of material which are in the vicinity of the detectors (e.g. re-etching of copper surfaces) and additional shielding. To reduce the crystalintrinsic backgrounds, the crystal production at TUM is key. We plan to significantly reduce 
the cosmogenic activation of $\mathrm{W}$ isotopes by using only screened and assayed materials, and to shorten the exposition to cosmic rays during transport and storage. It was found, that the growth procedure itself segregates certain radioactive isotopes significantly. Comparing the contamination of ${ }^{226} \mathrm{Ra}$ in TUM40 $((43.0 \pm 9.9) \mu \mathrm{Bq} / \mathrm{kg}$, see Table 2$)$ to that in the raw materials $(\mathcal{O}(10 \mathrm{mBq} / \mathrm{kg})[20])$, a purification by almost 3 orders of magnitude is achieved. Therefore, a multiple re-crystallization of $\mathrm{CaWO}_{4}$ crystals is a promising technique to decrease both, cosmogenic activation and contaminations from natural decay chains.

\section{Acknowledgments}

This research was supported by the DFG cluster of excellence: Origin and Structure of the Universe, the DFG Transregio 27: Neutrinos and Beyond, the Helmholtz Alliance for Astroparticle Phyiscs, the Maier-Leibnitz-Laboratorium (Garching), the STFC (United Kingdom) and by the BMBF: Project 05A11WOC EURECA-XENON. We are grateful to LNGS for their generous support of CRESST, in particular to Marco Guetti for his constant assistance.

\section{References}

[1] G. Bertone, D. Hooper, and J. Silk, Particle dark matter: Evidence, candidates and constraints, Phys.Rept. 405 (2005) 279-390, [hep-ph/0404175].

[2] G. Jungman, M. Kamionkowski, and K. Griest, Supersymmetric dark matter, Phys.Rept. 267 (1996) 195-373, [hep-ph/9506380].

[3] LUX Collaboration Collaboration, D. Akerib et al., First results from the LUX dark matter experiment at the Sanford Underground Research Facility, Phys.Rev.Lett. 112 (2014) 091303, [arXiv: 1310.8214].

[4] P. Cushman et al., Snowmass CF1 Summary: WIMP Dark Matter Direct Detection, arXiv: 1310.8327.

[5] DAMA, LIBRA Collaboration, R. Bernabei et al., New results from DAMA/LIBRA, Eur.Phys.J. C67 (2010) 39-49, [arXiv:1002.1028].

[6] CoGeNT Collaboration Collaboration, C. Aalseth et al., Cogent: A search for low-mass dark matter using p-type point contact germanium detectors, Phys. Rev. D 88 (Jul, 2013) 012002.

[7] CDMS Collaboration Collaboration, R. Agnese et al., Silicon detector dark matter results from the final exposure of cdms ii, Phys. Rev. Lett. 111 (Dec, 2013) 251301.

[8] CRESST-II Collaboration Collaboration, G. Angloher et al., Results on low mass WIMPs using an upgraded CRESST-II detector, arXiv:1407.3146.

[9] XENON100 Collaboration, E. Aprile et al., Dark Matter Results from 225 Live Days of XENON100 Data, Phys.Rev.Lett. 109 (2012) 181301, [arXiv:1207.5988].

[10] SuperCDMS Collaboration Collaboration, R. Agnese et al., Search for Low-Mass WIMPs with SuperCDMS, Phys.Rev.Lett. 112 (2014) 241302, [arXiv:1402.7137].

[11] SuperCDMSSoudan Collaboration Collaboration, R. Agnese et al., CDMSlite: A Search for Low-Mass WIMPs using Voltage-Assisted Calorimetric Ionization Detection in the SuperCDMS Experiment, Phys.Rev.Lett. 112 (2014) 041302, [arXiv: 1309.3259].

[12] EDELWEISS Collaboration, E. Armengaud et al., A search for low-mass WIMPs with EDELWEISS-II heat-and-ionization detectors, Phys.Rev. D86 (2012) 051701, [arXiv: 1207.1815]. 
[13] A. Brown, S. Henry, H. Kraus, and C. McCabe, Extending the CRESST-II commissioning run limits to lower masses, Phys. Rev. D 85 (Jan, 2012) 021301.

[14] G. Angloher et al., Commissioning run of the CRESST-II dark matter search, Astropart.Phys. 31 (2009), no. $4270-276$.

[15] R. Strauss et al., A detector module with highly efficient surface-alpha event rejection operated in CRESST-II Phase 2, ArXiv e-prints, accepted for publication in Eur.Phys.J. C (2015) [arXiv: 1410.1753].

[16] J. Birks, The Theory and Practice of Scintillation Counting. Pergamon Press, 1964.

[17] R. Strauss et al., Energy-dependent light quenching in $\mathrm{CaWO}_{4}$ crystals at $\mathrm{mK}$ temperatures, Eur.Phys.J. C74 (2014), no. 7.

[18] CRESST Collaboration, G. Angloher et al., Results from $730 \mathrm{~kg}$ days of the CRESST-II Dark Matter search, Eur.Phys.J. C72 (2012), no. 4 1-22.

[19] A. Erb and J.-C. Lanfranchi, Growth of high-purity scintillating $\mathrm{CaWO}_{4}$ single crystals for the low-temperature direct dark matter search experiments CRESST-II and EURECA, CrystEngComm 15 (2013) 2301-2304.

[20] A. Münster et al., Radiopurity of $\mathrm{CaWO}_{4}$ crystals for direct dark matter search with CRESST and EURECA, J. Cosmol. Astropart. Phys. 5 (May, 2014) 18, [arXiv:1403.5114].

[21] R. F. Lang et al., Electron and gamma background in CRESST detectors, Astropart.Phys. 32 (Jan., 2010) 318-324, [arXiv:0905.4282].

[22] R. B. Firestone, C. M. Baglin, and S. Chu, Table of isotopes. No. Vol. 4. Wiley, 1999.

[23] R. Lang et al., Scintillator Non-Proportionality and Gamma Quenching in CaWO4, arXiv:0910.4414.

[24] C. Cozzini et al., Detection of the natural alpha decay of tungsten, Phys.Rev. C70 (2004) 064606, [nucl-ex/0408006].

[25] S. Agostinelli et al., Geant4 - a simulation toolkit, Nuclear Instruments and Methods in Physics Research Section A: Accelerators, Spectrometers, Detectors and Associated Equipment 506 (2003), no. $3250-303$.

[26] J. Allison et al., Geant4 developments and applications, Nuclear Science, IEEE Transactions on 53 (Feb, 2006) 270-278.

[27] J. Schmaler, The CRESST Dark Matter Search - New Analysis Methods and Recent Results. $\mathrm{PhD}$ thesis, TU München, 2010.

[28] G. Angloher et al., EURECA Conceptual Design Report, Physics of the Dark Universe 3 (2014), no. $041-74$.

[29] The CRESST Collaboration, G. Angloher, et al., Probing low WIMP masses with the next generation of CRESST detectors, ArXiv e-prints (Mar., 2015) [arXiv:1503.0806]. 
Table 2. Radioactive decays (alpha and beta) of the natural decay chains $\left({ }^{238} \mathrm{U},{ }^{235} \mathrm{U}\right.$ and $\left.{ }^{232} \mathrm{Th}\right)$ from intrinsic contamination observed with the crystal TUM40. The first $29 \mathrm{~kg}$-days of exposure of CRESST-II Phase 2 were used for the analysis. The Q-values $\left(E_{l i t}\right)$ from literature [22] of the individual reactions are compared with the energies $\left(E_{o b s}\right)$ observed in the detector. The corresponding activities $A$ are given with $1 \sigma$ errors. The branching ratios $(b r)$ of the individual reactions are listed. The symbol (a) indicates beta decays which cannot be identified individually but are in equilibrium with short-lived alpha decays. Alpha-lines marked with (b) have a significant overlap with stronger alpha sources. The symbol (c) indicates fast decays which cannot (or only partly) be distinguished from the proceeding decay within detector resolution. The total activity of the identified intrinsic alpha sources is $A_{t o t, \alpha}=3.08 \pm 0.04 \mathrm{mBq} / \mathrm{kg}$.

\begin{tabular}{|c|c|c|c|c|c|c|c|}
\hline chain & parent & mode & $b r[\%]$ & half-life & $E_{\text {lit }}[\mathrm{keV}]$ & $E_{o b s}[\mathrm{keV}]$ & $A[\mu \mathrm{Bq} / \mathrm{kg}]$ \\
\hline \multirow[t]{14}{*}{${ }^{238} \mathrm{U}$} & ${ }^{238} \mathrm{U}$ & $\alpha$ & 100 & $4.47 \cdot 10^{9} \mathrm{y}$ & 4270 & 4271 & $(1.01 \pm 0.02) \cdot 10^{3}$ \\
\hline & ${ }^{234} \mathrm{Th}$ & $\beta^{-}$ & 100 & $24.1 \mathrm{~d}$ & 273 & $0-273$ & (a) \\
\hline & ${ }^{234} \mathrm{~Pa}^{*}$ & $\beta^{-}$ & 100 & $1.17 \mathrm{~min}$ & 2197 & $0-2197$ & (a) \\
\hline & ${ }^{234} \mathrm{U}$ & $\alpha$ & 100 & $2.45 \cdot 10^{5} \mathrm{y}$ & 4858 & 4853 & $(1.08 \pm 0.03) \cdot 10^{3}$ \\
\hline & ${ }^{230} \mathrm{Th}$ & $\alpha$ & 100 & $75.4 \mathrm{y}$ & 4770 & 4771 & $55.8 \pm 5.4$ \\
\hline & ${ }^{226} \mathrm{Ra}$ & $\alpha$ & 100 & $1.60 \mathrm{y}$ & 4871 & $4853(\mathrm{~b})$ & $43.0 \pm 9.9$ \\
\hline & ${ }^{222} \mathrm{Rn}$ & $\alpha$ & 100 & $3.82 \mathrm{~d}$ & 5590 & 5592 & $38.1 \pm 4.9$ \\
\hline & ${ }^{218} \mathrm{Po}$ & $\alpha$ & 99.98 & $3.10 \mathrm{~min}$ & 6115 & 6139 & $43.1 \pm 9.9$ \\
\hline & ${ }^{214} \mathrm{~Pb}$ & $\beta^{-}$ & 100 & $26.8 \mathrm{~min}$ & 1023 & 0-1023 & (a) \\
\hline & ${ }^{214} \mathrm{Bi}$ & $\beta^{-}$ & 99.98 & $19.9 \mathrm{~min}$ & 3272 & \multirow{2}{*}{ 7800-11000 } & \multirow{2}{*}{$47.4 \pm 4.9$} \\
\hline & ${ }^{214} \mathrm{Po}$ & $\alpha$ & 100 & $0.164 \mathrm{~ms}(\mathrm{c})$ & 7883 & & \\
\hline & ${ }^{210} \mathrm{~Pb}$ & $\beta^{-}$ & 100 & $22.3 \mathrm{y}$ & 63.5 & $0-63.5$ & $7_{-7}^{+36}$ \\
\hline & ${ }^{210} \mathrm{Bi}$ & $\beta^{-}$ & 100 & $5.01 \mathrm{~d}$ & 1163 & $0-1163$ & (a) \\
\hline & ${ }^{210} \mathrm{Po}$ & $\alpha$ & 100 & $138 \mathrm{~d}$ & 5407 & 5403 & $17.8 \pm 4.0$ \\
\hline \multirow[t]{11}{*}{${ }^{235} \mathrm{U}$} & ${ }^{235} \mathrm{U}$ & $\alpha$ & 100 & $7.04 \cdot 10^{8} \mathrm{y}$ & 4678 & 4671 & $39.5 \pm 4.4$ \\
\hline & ${ }^{231} \mathrm{Th}$ & $\beta^{-}$ & 100 & $25.52 \mathrm{~h}$ & 389.5 & $0-389.5$ & (a) \\
\hline & ${ }^{231} \mathrm{~Pa}$ & $\alpha$ & 100 & $3.27 \cdot 10^{4} \mathrm{y}$ & 5150 & 5139 & $23.2 \pm 4.4$ \\
\hline & ${ }^{227} \mathrm{Ac}$ & $\beta^{-}$ & 98.62 & $21.8 \mathrm{y}$ & 44.8 & $0-44.8$ & $98 \pm 20$ \\
\hline & ${ }^{227} \mathrm{Th}$ & $\alpha$ & 100 & $18.7 \mathrm{~d}$ & 6147 & 6139 & $105 \pm 19$ \\
\hline & ${ }^{223} \mathrm{Ra}$ & $\alpha$ & 100 & $11.4 \mathrm{~d}$ & 5979 & 5968 & $104 \pm 7$ \\
\hline & ${ }^{219} \mathrm{Rn}$ & $\alpha$ & 100 & $3.96 \mathrm{~s}$ & 6946 & \multirow{2}{*}{14900} & \multirow{2}{*}{$107 \pm 7$} \\
\hline & ${ }^{215} \mathrm{Po}$ & $\alpha$ & 100 & $1.78 \mathrm{~ms}(\mathrm{c})$ & 7527 & & \\
\hline & ${ }^{211} \mathrm{~Pb}$ & $\beta^{-}$ & 100 & $36.1 \mathrm{~min}$ & 1373 & $0-1373$ & (a) \\
\hline & ${ }^{211} \mathrm{Bi}$ & $\alpha$ & 100 & $0.51 \mathrm{~s}$ & 6751 & 6771 & $105 \pm 7$ \\
\hline & ${ }^{207} \mathrm{Tl}$ & $\beta^{-}$ & 100 & $4.77 \mathrm{~min}$ & 1423 & $0-1423$ & (a) \\
\hline \multirow[t]{12}{*}{${ }^{232} \mathrm{Th}$} & ${ }^{232} \mathrm{Th}$ & $\alpha$ & 100 & $1.40 \cdot 10^{10} \mathrm{y}$ & 4083 & 4084 & $9.2 \pm 2.3$ \\
\hline & ${ }^{228} \mathrm{Ra}$ & $\beta^{-}$ & 100 & $5.75 \mathrm{y}$ & 45.9 & $6.7-45.9$ & (a) \\
\hline & ${ }^{228} \mathrm{Ac}$ & $\beta^{-}$ & 100 & $6.15 \mathrm{~h}$ & 2127 & $58-2127$ & (a) \\
\hline & ${ }^{228} \mathrm{Th}$ & $\alpha$ & 100 & $1.91 \mathrm{y}$ & 5520 & 5518 & $15.2 \pm 4.1$ \\
\hline & ${ }^{224} \mathrm{Ra}$ & $\alpha$ & 100 & $3.63 \mathrm{~d}$ & 5789 & 5788 & $19.8 \pm 8.1$ \\
\hline & ${ }^{220} \mathrm{Rn}$ & $\alpha$ & 100 & $55.6 \mathrm{~s}$ & 6404 & 6414 & $8.4 \pm 3.4$ \\
\hline & ${ }^{216} \mathrm{Po}$ & $\alpha$ & 100 & $0.145 \mathrm{~s}$ & 6906 & - & 0 \\
\hline & ${ }^{212} \mathrm{~Pb}$ & $\beta^{-}$ & 100 & $10.64 \mathrm{~h}$ & 573.7 & $0-573.7$ & (a) \\
\hline & ${ }^{212} \mathrm{Bi}$ & $\alpha$ & 35.94 & $60.6 \mathrm{~min}$ & 6208 & 6216 & $7.7_{-7.7}^{+8.9}$ \\
\hline & & $\beta^{-}$ & 64.06 & & 2252 & \multirow{2}{*}{ 8900-11200 } & \multirow{2}{*}{$15.8 \pm 2.8$} \\
\hline & ${ }^{212} \mathrm{Po}$ & $\alpha$ & 100 & $299 \mathrm{~ns}(\mathrm{c})$ & 8955 & & \\
\hline & ${ }^{208} \mathrm{Tl}$ & $\beta^{-}$ & 100 & $3.01 \mathrm{~min}$ & 5001 & $3197-5001$ & (a) \\
\hline
\end{tabular}


Table 3. Activities $\left(A_{1-40}\right)$ of the identified background sources in the $e^{-} / \gamma$-band between 1 and $40 \mathrm{keV}$ and the expected leakage $L_{1}\left(L_{12}\right)$ per detector $(m=245 \mathrm{~g})$ and year into the region-of-interest (ROI) assuming an analysis threshold of $1 \mathrm{keV}(12 \mathrm{keV})$. The activities of the relevant $\beta^{-}$-decaying isotopes from natural decay chains are derived by a MC-based analysis (see text). The sum of all intrinsic beta decays $(\Sigma$ internal $\beta / \gamma)$, of the cosmogenic activation lines ( $\Sigma$ cosmogenics), of the external $\gamma$ radiation $(\Sigma$ external $\gamma)$ and of the external $\beta$ radiation $(\Sigma$ external $\beta)$ are listed. The sum of all identified sources is compared to the activity observed with in the data of CRESST-II Phase 2. For $L_{1}$ and $L_{12}$ the total errors are of $\mathcal{O}(10 \%)$. (a) indicates an extrapolation to the assumed exposure of 1 detector-year.

\begin{tabular}{lccc}
\hline source & $A_{1-40}[\mu \mathrm{Bq} / \mathrm{kg}]$ & $L_{1} /[$ det.-y $]$ & $L_{12} /[$ det.-y] \\
\hline${ }^{234} \mathrm{Th}$ & $346.2 \pm 12.3$ & 18.1 & $2.0 \cdot 10^{-5}$ \\
${ }^{234} \mathrm{~Pa}^{*}$ & $35.0 \pm 5.7$ & 1.6 & $2 \cdot 10^{-6}$ \\
${ }^{214} \mathrm{~Pb}$ & $(8.3 \pm 0.4) \cdot 10^{-1}$ & $3.7 \cdot 10^{-2}$ & $<10^{-6}$ \\
${ }^{210} \mathrm{~Pb}$ & $1.0 \pm 0.1$ & $8.6 \cdot 10^{-2}$ & $<10^{-6}$ \\
${ }^{210} \mathrm{Bi}$ & $(3.6 \pm 0.4) \cdot 10^{-1}$ & $1.6 \cdot 10^{-2}$ & $<10^{-6}$ \\
${ }^{231} \mathrm{Th}$ & $1.1 \cdot 10^{-2}$ & $2.5 \cdot 10^{-4}$ & $<10^{-6}$ \\
${ }^{227} \mathrm{Ac}$ & $92.9 \pm 1.8$ & 5.6 & $9.0 \cdot 10^{-6}$ \\
${ }^{211} \mathrm{~Pb}$ & $4.2 \pm 0.1$ & $1.9 \cdot 10^{-1}$ & $<10^{-6}$ \\
${ }^{207} \mathrm{Tl}$ & $3.9 \pm 0.6$ & $1.7 \cdot 10^{-1}$ & $<10^{-6}$ \\
${ }^{228} \mathrm{Ra}$ & $8.9 \pm 0.2$ & $8.3 \cdot 10^{-4}$ & $1 \cdot 10^{-6}$ \\
${ }^{212} \mathrm{~Pb}$ & $2.4 \pm 0.4) \cdot 10^{-1}$ & $1.1 \cdot 10^{-2}$ & $<10^{-6}$ \\
$\mathrm{Cu} \mathrm{X}$-ray $($ ext. $)$ & $27.1 \pm 5.1$ & $4.4 \cdot 10^{-4}$ & - \\
\hline$\Sigma$ internal $\beta / \gamma$ & $494.2 \pm 48.4$ & 25.7 & $3.2 \cdot 10^{-5}$ \\
$\Sigma$ cosmogenics & $290.7 \pm 17.0$ & 40.8 & $<10^{-6}$ \\
$\Sigma$ external $\gamma$ & $62.2 \pm 7.8$ & $3.9 \cdot 10^{-1}$ & $1 \cdot 10^{-6}$ \\
$\Sigma$ external $\beta$ & $274.7 \pm 137.6$ & 7.8 & $1.6 \cdot 10^{-5}$ \\
$\Sigma$ all identified & $1121.8 \pm 146.6$ & 74.7 & $4.9 \cdot 10^{-5}$ \\
\hline total observed & $1625.1 \pm 40.3$ & $108.3(\mathrm{a})$ & $7.1 \cdot 10^{-5}(\mathrm{a})$ \\
\hline
\end{tabular}

